\title{
Fatores de risco para hipoglicemia em pacientes que usam infusão contínua de insulina endovenosa na unidade de terapia intensiva
}

\author{
Risk factors for hypoglycemia in patients using continuous \\ infusion of insulin in intensive care \\ Carina Teixeira Paixão, ${ }^{1,2}$, Lolita Dopico da Silva ${ }^{1,3}$, Paula Borba Doerzapff ${ }^{4}$, Raquel Magalhães de Azeredo Granadeiro ${ }^{4}$, \\ Renata Lemos de Aragão Farias ${ }^{3,5}$, Sheyla Silva Santos ${ }^{4}$ \\ ${ }^{1}$ Curso de Especialização de Enfermagem Intensivista da Faculdade de Enfermagem da Universidade do Estado do Rio de Janeiro (FEUERJ) - Rio de Janeiro (RJ), Brasil. \\ 2Departamento de Rotina do Pós-operatório de Cirurgia Cardíaca do Instituto Nacional de Cardiologia (INC) - Rio de Janeiro (RJ), Brasil. \\ ${ }^{3}$ Programa de Pós-Graduação de Enfermagem Intensivista da FEUERJ - Rio de Janeiro (RJ), Brasil. \\ 4Pós-Graduação em Terapia Intensiva da FEUERJ - Rio de Janeiro (RJ), Brasil. \\ ${ }^{5}$ Departamento de Rotina da Unidade de Terapia Intensiva do Hospital Estadual Roberto Chabo (HERC) - Araruama (RJ), Brasil.
}

DOI: http://dx.doi.org/10.7322/abcshs.v39i3.655

\section{RESUMO}

Trata-se de revisão descritiva dos fatores de risco para hipoglicemia em pacientes com infusão contínua de insulina na terapia intensiva. Buscaram-se publicações entre 2006 e 2012, em livros e artigos de bases indexadas em bibliotecas virtuais. Selecionadas oito publicações ordenadas em categorias que tratavam da hipoglicemia, terapia nutricional e protocolos glicêmicos associados à insulinoterapia venosa. Conclui-se que há fatores de risco comuns à equipe multiprofissional e o conhecimento desses fatores permitirá a segurança dos pacientes na utilização dessa terapêutica.

Palavras-chave: hipoglicemia; fatores de risco; cuidados de enfermagem; terapia intensiva.

\begin{abstract}
This work is a descriptive review on risk factors for hypoglycemia in patients with continuous insulin infusion in intensive care unit. Publications from 2006 to 2012 were searched in books and articles of indexed databases from virtual libraries. Eight publications were selected and sorted into categories that addressed hypoglycemia, nutritional therapy, and glycemic protocols associated with intravenous insulin therapy. It is concluded that there are common risk factors for the multidisciplinary team and the knowledge of these factors will allow patient safety for using this therapy.
\end{abstract}

Keywords: hypoglycemia; risk factors; nursing care; intensive care. 


\section{INTRODUÇÃO}

Esta pesquisa apresenta como objeto de estudo fatores de risco para hipoglicemia em pacientes com infusão contínua de insulina.

Pacientes internados em unidades de terapia intensiva (UTI) estão sob estado de estresse fisiológico em virtude da gravidade de seu quadro clínico, predispondo-os a diversos distúrbios endócrinos, tais como a hiperglicemia. Foi identificada hiperglicemia em $38 \%$ dos pacientes cirúrgicos admitidos em UTI, dos quais um terço não tinha história de diabetes prévia à admissão ${ }^{1}$.

Os fatores que cooperam para hiperglicemia nesses pacientes incluem a liberação de hormônios de estresse (epinefrina, glucagon, hormônio do crescimento e cortisol) e o uso de medicações, como corticoides e catecolaminas. Nos casos de sepse ou trauma cirúrgico, há a liberação de citocinas inflamatórias, acarretando alterações hemodinâmicas, distúrbio eletrolítico, desidratação, estresse oxidativo, aumento de fatores inflamatórios, hipercoagulabilidade, piora de isquemia miocárdica e cerebral, alteração de imunidade, retardo na cicatrização, inflamação e piora da função endotelial ${ }^{1}$.

A hiperglicemia em pacientes críticos apresenta um maior risco de complicações relacionadas ao sistema inflamatório, além de um aumento no tempo de internação hospitalar e nos índices de mortalidade ${ }^{2}$. É notório que a hiperglicemia contribui para a elevação da gravidade da doença ${ }^{1}$. Portanto, a manutenção da normoglicemia está associada a taxas reduzidas de infecções e de falências orgânicas, podendo levar a uma menor mortalidade ${ }^{3}$.

Níveis glicêmicos elevados alteram também a função imunológica e o controle glicêmico adequado melhora a função de macrófagos/neutrófilos. Por isso, a redução da glicemia pela infusão venosa contínua de insulina passou a ser o tratamento utilizado para esse tipo de paciente ${ }^{3}$.

Para pacientes críticos, as metas de níveis glicêmicos variam de 140 a $180 \mathrm{mg} / \mathrm{dL}$, evitando glicemias abaixo de $100 \mathrm{mg} / \mathrm{dL}$. Considera-se hipoglicemia moderada os valores de $70 \mathrm{mg} / \mathrm{dL}$ e hipoglicemia grave aqueles abaixo de $40 \mathrm{mg} / \mathrm{dL}$. A monitorização desses valores é primordial e deve obedecer tanto os níveis absolutos como também as oscilações glicêmicas. Geralmente, inicia-se a infusão contínua de insulina nos valores acima de $180 \mathrm{mg} / \mathrm{dL}$. Durante a infusão endovenosa de insulina, o ideal é medir a glicemia a cada hora, sendo possível aumentar esse intervalo para cada duas horas ou três horas, nos pacientes com controle glicêmico dentro da meta estabelecida para cada unidade hospitalar ${ }^{1}$.

A hipoglicemia apresenta-se como a principal complicação associada à infusão contínua de insulina utilizada para o controle glicêmico. Dentre os principais motivos encontramos: excesso de insulina administrada, deficiência na resposta ao glucagon e à epinefrina, rebaixamento do nível de consciência (sedação ou outras causas clínicas), deficiências hormonais, fármacos associados e interrupção do suporte nutricional ${ }^{4}$.

As manifestações clínicas são: sudorese, tremor, taquicardia, palpitação, nervosismo e fome, caracterizando a hipoglicemia branda. A moderada apresenta sinais de comprometimento do sistema nervoso central, podendo incluir a incapacidade de se concentrar, cefaleia, tontura, confusão e lapsos de memória.

Já na hipoglicemia grave, o paciente apresenta desorientação, convulsões, dificuldade de despertar do sono ou perda da consciência ${ }^{5}$. É necessária a correção imediata dessa hipoglicemia antes que cause dano celular ${ }^{1}$.

Estudos publicados como o de van den Berghe et al. ${ }^{5}$ reafirmam que a hipoglicemia é o efeito adverso mais importante com o uso da insulina endovenosa na terapia intensiva. Essa intervenção aumentou em seis vezes a incidência de hipoglicemia. E apesar de não ter havido óbito relacionado a tal terapia, a hipoglicemia pode causar dano e, portanto, foi identificada como um fator independente de risco letal nos pacientes clínicos de $\mathrm{UTI}^{5}$. Já nas unidades coronarianas, a hipoglicemia estimula o sistema simpático, propiciando a ocorrência de arritmias e aumentando a demanda miocárdica de oxigênio, favorecendo o aparecimento de quadros anginosos ${ }^{4}$.

Por isso, é fundamental que o enfermeiro reconheça e evite os fatores que levam a essa disfunção, uma vez que esse é o profissional responsável pela verificação da glicemia, manutenção e ajustes da insulina venosa dentro da UTI.

Diante do exposto, o objetivo deste trabalho foi levantar na literatura artigos relacionados aos fatores de risco para hipoglicemia nos pacientes que utilizam infusão contínua de insulina.

\section{MÉTODOS}

Esta pesquisa classifica-se como revisão descritiva com as seguintes etapas: definição da questão norteadora, seleção dos descritores, definição do material bibliográfico, critérios de seleção, levantamento do material bibliográfico, organização das categorias e análise dos dados obtidos. O estudo foi guiado pela seguinte questão norteadora: Quais os fatores de risco para hipoglicemia em pacientes que utilizam infusão contínua de insulina?

A identificação do objeto de estudo foi realizada incluindo todos os artigos publicados no período de 2005 a 2012 e indexados na Biblioteca Virtual da Saúde (BVS), Literatura LatinoAmericana e do Caribe em Ciências da Saúde (LILACS), Scientific Electronic Library Online (SciELO), Índice Bibliográfico Espanhol de Ciências da Saúde (IBECS), Literatura Internacional em Ciências da Saúde (MEDLINE) e PubMed. Os critérios de seleção foram artigos em português, inglês e espanhol com textos disponíveis na íntegra no período estabelecido e indexados pelos termos dos Descritores em Ciências da Saúde (DeCS) com os seguintes descritores: hipoglicemia, fatores de risco, cuidados de enfermagem e terapia intensiva; referindo-se à população adulta hospitalizada e que abordassem os fatores de risco para hipoglicemia em pacientes com infusão contínua de insulina endovenosa.

A busca foi realizada no período de julho a novembro de 2012. Para a coleta de dados foi elaborado um instrumento baseado em 
protocolos de revisão bibliográfica sendo substituídos os itens do original "citation" e "intervention" por títulos e procedimentos metodológicos. Os resultados foram agrupados em categorias, o que norteou a discussão do estudo.

\section{RESULTADOS E DISCUSSÃO}

Foram encontrados 157 artigos e selecionadas 8 publicações que compuseram três categorias: os que se referem à hipoglicemia associada à infusão contínua de insulina; à terapia nutricional e infusão de insulina; e ao controle glicêmico durante a insulinoterapia endovenosa.

\section{Hipoglicemia associada à insulinoterapia endovenosa}

Faust et al. ${ }^{6}$ enfatizaram as nuances dos escritos e pequisas sobre controle glicêmico na terapia intensiva e propuseram uma recomendação para tal controle. Relataram que a prática da insulina infundida por via subcutânea era inapropriada, pois os valores de glicose sanguínea ficavam acima de $180 \mathrm{mg} / \mathrm{dL}$ e isso estava associado a resultados negativos. Pacientes deveriam ser mais bem manejados usando taxas glicêmicas que afastassem a variação da glicose sanguínea. O alvo da glicemia ajustada para valores maiores poderia reduzir o número de episódios de hipoglicemia severa, com diminuição da mortalidade. Ao analisar a relação da hipoglicemia leve ou moderada sob a mortalidade de pacientes graves, relataram que dos 4.946 pacientes internados na UTI, 1.109 tinham pelo menos um episódio de hipoglicemia $(<81 \mathrm{mg} / \mathrm{dL})^{6}$. Verificou-se que em pacientes criticamentes enfermos existe uma associação entre a hipoglicemia e a mortalidade, e quanto maior grau de hipoglicemia maior o risco de morte ${ }^{6}$.

Lacherade et al. ${ }^{7}$ analisaram a hipoglicemia em pacientes críticos e seus efeitos em diferentes órgãos, a maneira como era realizada a mensuração e o controle da glicemia e o impacto na mortalidade e morbimortalidade. Dentre as principais causas de hipoglicemia, é citado o inadequado suporte nutricional, doença grave que limita a produção de glicose ou acelera sua utilização. Além disso, observou-se que manter a normoglicemia é um processo complexo, pois envolve medições de glicemia, leitura de protocolos, registros, mudanças na dose de insulina e supervisão de nutrição ${ }^{7}$. Por isso, a equipe multiprofissional é fundamental. Logo, a incapacidade de seguir protocolos, a sobrecarga de trabalho e eventuais erros são riscos adicionais para hipoglicemia. Destacam-se outros fatores que contribuem para hipoglicemia, como a insuficiência renal e/ou hepática, insuficiência supra-renal e o tratamento com quinolona ${ }^{7}$.

Egi et al. ${ }^{8}$ avaliaram os riscos e benefícios do tratamento intensivo de insulina (TII) em diferentes instituições com pacientes em pós-operatório com características clínicas e demográficas semelhantes e em ventilação mecânica. Foram medidos os níveis de glicose em todos os pacientes. A hipoglicemia foi definida como 2,2 mmol/L (36 mg/dL). Concluíram que o risco de hipoglicemia associado ao TII pode variar bastante de acordo com o grau de gravidade da doença e da doença que acomete o paciente, sendo importante avaliar os possíveis benefícios e os riscos do tratamento antes de sua implementação ${ }^{8}$.

É notório que a hipoglicemia é considerada a principal complicação associada à infusão de insulina na terapia intensiva, ocorrendo em torno de 2 a $11 \%$ dos pacientes ${ }^{9}$. Em 2006, UTI mistas avaliaram 2.748 pacientes clínicos e cirúrgicos e verificaram hipoglicemia em 11,3\% dos pacientes que utilizavam insulina endovenosa. Foram considerados fatores de risco para hipoglicemia nesse grupo a sepse, a falência orgânica e a necessidade de hemodiálise ${ }^{9}$.

Reconhecer os fatores de risco é muito importante para evitar a hipoglicemia. Os fatores mais importantes são: gravidade da doença definida pelo escore Acute Physiologic Health Evaluation II (APACHE II), presença de insuficiência renal (retardo no clearance de insulina), sepse e insuficiência hepática, descontinuidade de calorias com a infusão contínua de insulina e diabetes mellitus. Pacientes com choque séptico parecem estar em um maior risco para desenvolver hipoglicemia ${ }^{10}$. Graves reações hipoglicêmicas como letargia, convulsões e inconsciência surgem quando os níveis glicêmicos encontram-se menores que $40 \mathrm{mg} / \mathrm{dL}$, o que compromete a vida do paciente, podendo levar ao óbito ou provocar danos neurológicos cumulativos e permanentes ${ }^{11}$.

Terapia nutricional e infusão de insulina

Gunst e van den Berghe G. ${ }^{12}$ analisaram os estudos sobre infusão contínua de insulina. Compararam o Normoglycemia in Intensive Care Evaluation - Survival Using Glucose Algorithm Regulation (NICE-SUGAR) e o feito pela equipe médica do Hospital Universitário de Leuven, na Bélgica. Em Leuven, o resultado com a infusão contínua de insulina foi benéfico, já o estudo NICE-SUGAR encontrou um aumento no risco da mortalidade. Justificaram os resultados considerando que havia diferenças entre a medição de glicose e o regime de alimentação. Em Leuven, a nutrição enteral ou parenteral eram fornecidas imediatamente após a infusão contínua de insulina, enquanto que no estudo intitulado NICE-SUGAR, a mesma forma de nutrição era dada tardiamente após a infusão contínua de insulina. Concluíram que o alvo glicêmico ideal precisa ser definido e que há necessidade de se ter uma equipe multiprofissional bem treinada. A hipoglicemia e a hiperglicemia excessivas deveriam ser impedidas em todos os pacientes ${ }^{12}$.

O controle glicêmico é afetado diretamente pelo descontrole ou mau controle nutricional. Pacientes com alteração na capacidade de relatar sintomas, redução de ingestão oral, vômito, diminuição ou interrupção da dieta enteral ou parenteral apresentam maior risco para hipoglicemia. É fundamental o controle do peso, o estado nutricional e a verificação da oferta de glicose (dieta enteral, parenteral ou glicose contínua), evitando episódios de hiperglicemia ou hipoglicemia ${ }^{11}$. 
Essa avaliação nutricional deve fornecer calorias (carboidratos, lipídios, proteínas, água, vitaminas e eletrólitos) suficientes para esses pacientes com insulinoterapia endovenosa, além do controle glicêmico rigoroso, a fim de evitar eventos hipoglicêmicos ${ }^{13}$.

\section{Controle glicêmico durante a insulinoterapia}

Ellahham ${ }^{14}$ analisou os dados que estabelecem a ligação entre hiperglicemia e seus riscos de morbimortalidade, além de descrever estratégias que provam ser eficazes na manutenção da glicemia e seu controle em pacientes críticos. Os resultados obtidos foram: o valor da glicose no sangue convencional de $140-180 \mathrm{mg} / \mathrm{dL}$ foi considerado desejável e seguramente possível na maioria dos pacientes. O controle mais agressivo, a $110 \mathrm{mg} / \mathrm{dL}$, tem mostrado benefícios para pacientes em terapia intensiva cirúrgica, pois aumenta a sobrevida e diminuiu o tempo do paciente na UTI, porém, em pacientes que necessitam de um período superior a três dias de internação, esse nível glicêmico foi desfavorável aumentando a incidência de hipoglicemia ${ }^{14}$.

Vogelzang et al. ${ }^{15}$ avaliaram a eficácia do protocolo de controle de glicose computadorizado em pacientes criticamente enfermos, em estudo de coorte observacional em três unidades de terapia intensiva. Todos os 2.800 pacientes internados na neurocirurgia, pós-cirúrgicos e unidades cardiotorácicas foram submetidos à regulação da glicose através de um sistema computadorizado. Foram analisados o cumprimento em relação às taxas de insulina recomendados, bomba e frequência de medição de glicose. Esse protocolo de controle de glicose computadorizado fornece uma regulação segura e eficiente na prática de cuidados de rotina intensa. O baixo episódio de hipoglicemia foi alcançado com um número consideravelmente menor de medições de glicose do que utilizando outros métodos ${ }^{15}$.

Malesker et al. ${ }^{16}$ verificaram a eficiência de protocolos rígidos de controle glicêmico utilizado nas UTI. Perceberam que enfermeiros gastam tempo substancial para administrar protocolos de controle glicêmico rígido. Novos esforços educacionais e avaliação permanente do impacto de tais protocolos são necessários.

O controle glicêmico nas UTI é feito frequentemente com pequenas amostras de sangue em uma linha arterial, venosa ou capilar. Esse é um procedimento imprescindível e de substancial importância na avaliação do estado do paciente. Entretanto, esse controle glicêmico envolve diversos cuidados, como padronização de protocolos, treinamento do pessoal de enfermagem e utilização de equipamentos adequados a fim de evitar riscos excessivos de hipoglicemia e ineficácia do controle ${ }^{17}$. Em pacientes clínicos, o objetivo da utilização de insulina endovenosa é manter níveis glicêmicos normais que variam entre $80-110 \mathrm{mg} / \mathrm{dL}$, o que pode estar associado à ocorrência de hipoglicemia ${ }^{11}$.

A monitorização da infusão de insulina envolve medidas frequentes de glicemia capilar para conseguir alcançar as faixas glicêmicas alvo e prevenir efeitos adversos relacionados à hipoglicemia. Os métodos utilizados para medida da glicemia à beira leito devem ser seguros e acurados. É importante que haja uma avaliação com cautela em relação ao modo de processamento e a coleta de sangue arterial, venoso central ou capilar. A glicemia verificada através da coleta digital, ou seja por dígito-pressão em pacientes hemodinamicamente instáveis pode ser inexata, devido ao prejuízo da perfusão periférica ocasionado pelo uso de drogas vasoativas, presença de edema e distúrbios na microcirculação, podendo levar a modificações inapropriadas na vazão da infusão de insulina ${ }^{18}$.

A enfermagem é peculiar no manejo de distúrbios glicêmicos, com cuidados que incluem vigilância do paciente referente aos sinais de hiper e hipoglicemia, preparo e infusão da solução de insulina, monitorização laboratorial, hídrica, nutricional e controle da glicemia ${ }^{10}$.

Vale salientar que a glicemia ideal é aquela controlada o mais precocemente possível, com uso da menor dose de insulina para evitar possíveis efeitos deletérios do excesso de insulina e manter apenas os efeitos benéficos.

\section{CONSIDERAÇÕES FINAIS}

A hipoglicemia é considerada a principal complicação associada à infusão de insulina na terapia intensiva. Dentre os fatores de risco para hipoglicemia destacamos: não padronização da via de coleta do sangue e atrasos nas aferições da glicemia, ajustes inadequados da velocidade de infusão da insulina endovenosa descritos nos protocolos glicêmicos e ausência ou inadequado suporte nutricional. Foram evidenciados outros fatores como a sepse, falência orgânica, insuficiência renal com necessidade de hemodiálise, doença hepática e diabetes mellitus.

Outro aspecto que se destaca através desta revisão é que não há publicações com essa temática por parte dos enfermeiros, o que abre um amplo leque de possibilidades de pesquisas feitas por enfermeiros.

Não foram encontrados artigos que abordem os diversos fatores que podem interferir no procedimento do controle glicêmico, feito por enfermeiros. Esses devem pensar na possiblidade de realizar estudos sobre a influência desses diferentes fatores de risco nas taxas glicêmicas e que cuidados de enfermagem específicos podem ser adotados para evitar a hipoglicemia junto aos pacientes que tenham fatores de risco presentes na sua condição clínica. 


\section{REFERÊNCIAS}

1. Sociedade Brasileira de Diabetes. Controle da hiperglicemia intrahospitalar em pacientes críticos e não críticos. Posicionamento Oficial SBD no 02/2011. Disponível em: http://www.nutritotal.com. br/diretrizes/files/228--posicionamento_sbd_hiperglicemia.pdf. Acesso em: 20 jan. 2013.

2. Pasquel FJ, Umpierrez GE. Manejo de la hiperglucemia en el paciente hospitalizado. Medicina (B. Aires). 2011;7(3):275-83.

3. Lazzari CM, Volkart T. Eficiência da solução de insulina: comparação entre diferentes tempos de manutenção da solução. Rev Bras Ter Intensiva. 2010;22(4):358-62. http://dx.doi.org/10.1590/S0103-507X2010000400008

4. Brunner LS, Suddarth DS. Tratado de Enfermagem Médico Cirúrgico. In: Brunner LS. Histórico e Cuidados aos Pacientes com Diabetes Melito. 10 ed. Rio de Janeiro: Guanabara Koogan; 2009. $1192 \mathrm{p}$.

5. Van den Berghe G, Wilmer A, Hermans G, Meersseman W, Wouters PJ, Milants I, et al. Intensive insulin therapy in the medical ICU. N Engl J Med. 2006:354(5):449-61. http://dx.doi.org/10.1056/NEJMoa052521

6. Faust AC, Attridge RL, Ryan L. How low should you go? The limbo of glycemic control in intensive care units. Crit Care Nurse. 2011;31(4):e9-18. http://dx.doi.org/10.4037/ccn2011188

7. Lacherade JC, Jacqueminet S, Preiser JC. An overview of hypoglycemia in the critically ill. J Diabetes Sci Technol. 2009;3(6):1242-9. http://dx.doi.org/10.1177/193229680900300603

8. Egi M, Bellomo R, Stachowski E, French CJ, Hart GK, Taori G, et al. Hypoglycemia and outcome in critically ill patients. Mayo Clin Proc. 2010;85(3):217-24.

http://dx.doi.org/10.4065/mcp.2009.0394

9. Egi M, Bellomo R, Stachawski E, French CJ, Hart G, Stow P, et al. Intensive insulin therapy in postoperative intensive care unit patients: a decision analysis. Am J Respir Crit Care Med. 2006;173(4):407-13. http://dx.doi.org/10.1164/rccm.200506-9610C
10. Associação de Medicina Intensiva Brasileira. Sociedade Brasileira de Infectologia. Instituto Latino Americano de Sepse. Sepse: Controle Glicêmico. In: Associação Médica Brasileira e Agência Nacional de Saúde Suplementar. Diretrizes Clínicas na Saúde Suplementar AMBANS; 2011. Disponivel em: http://www.projetodiretrizes.org.br/ ans/diretrizes/sepse-controle_glicemico.pdf. Acesso em: 20 jan. 2013.

11. NICE-SUGAR Study Investigators, Finfer S, Chittock DR, Su SY Blair D, Foster D, et al. Intensive versus conventional glucose control in critically ill patients. N Engl J Med. 2009;360(1):1283-97. http://dx.doi.org/10.1056/NEJMoa0810625

12. Gunst J, van den Berghe G. Blood glucose control in the intensive care unit: benefits and risks. Semin Dial. 2010;23(2):157-62. http://dx.doi.org/10.1111/j.1525-139X.2010.00702.x

13. Ferreira IKC. Terapia nutricional em Unidade de Terapia Intensiva Rev Bras Ter Intensiva. 2007;19(1):90-7 http://dx.doi.org/10.1590/S0103-507X2007000100012

14. Ellahham S. Insulin therapy in critically ill patients. Vasc Health Risk Manag. 2010;6:1089-101. http://dx.doi.org/10.2147/VHRM.S14203

15. Vogelzang M, Loef BG, Regtien JG, van der Horst IC, van Assen $\mathrm{H}$, Zijlstra F, et al. Computer-assisted glucose control in critically ill patients. Intensive Care Med. 2008;34(8):1421-7. http://dx.doi.org/10.1007/s00134-008-1091-y

16. Malesker MA, Foral PA, McPhillips AC, Christensen KJ, Chang JA Hilleman DE. An efficiency evaluation of protocols for tight control in intensive care units. Am J Crit Care. 2007;16(6):589-98.

17. Silva GG. Diabete $x$ UTI. Monografia (Curso de Especialização) Associação de Medicina Intensiva Brasileira, Cuiabá; 2011. Disponivel em: http://www.redentor.inf.br/arquivos/pos/ publicacoes/13082012MONOGRAFIA\%20DIABETE\%20x\%20 UTI. pdf. Acesso em: 10 dez. 2012.

18. Argollo APB, Faustino TN, Faustino TN, Pedreira LC. Valores glicêmicos oferecidos pelo glicosímetro portátil, utilizando sangue de diferentes vias de coletas: estudo de validade. Rev Bras Ter Intensiva. 2010;22(4):351-7. http://dx.doi.org/10.1590/S0103-507X2010000400007 